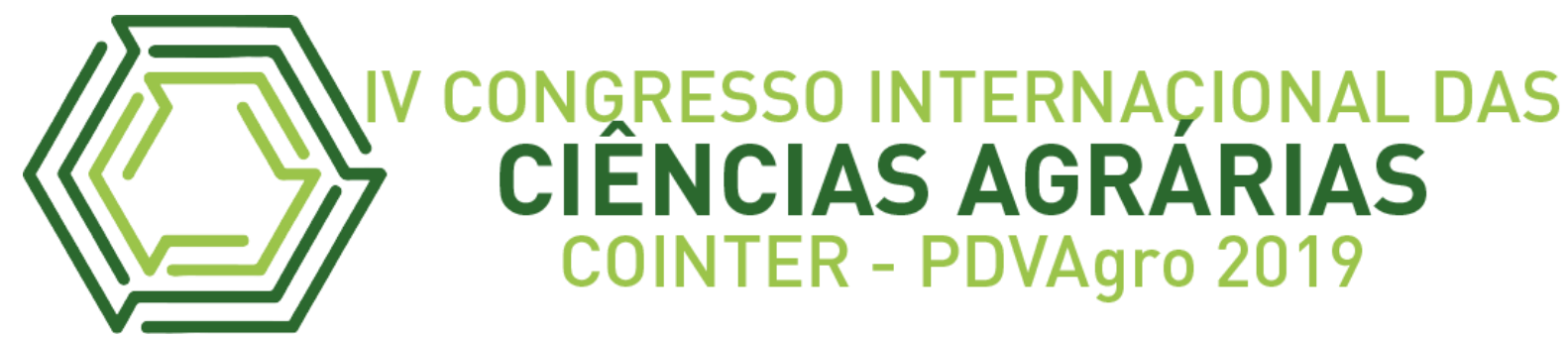

\title{
ANÁLISE DE DADOS DA CIÊNCIA DO SOLO NO R: ESTUDO DE CASO DAS TRANSFORMAÇÕES LOGARÍTMICAS
}

\section{ANÁLISIS DE DATOS DE LA CIENCIAS DEL SUELO EN R: ESTUDIO DE CASO DE LA TRANSFORMACIONES LOGARÍTICAS}

\section{SOIL SCIENCE DATA ANALYSIS IN THE R: CASE STUDY OF LOGARITIC TRANSFORMATIONS}

\author{
Apresentação: Comunicação Oral \\ Wanderson Benerval de Lucena ${ }^{1}$; Anderson Lucas da Silva ${ }^{2}$; José Lucas Farias da Silva ${ }^{3}$ \\ Hugo Isaac da Silva ${ }^{4}$; Marciano De Carvalho Batista ${ }^{5}$
}

DOI: $\underline{\text { https://doi.org/10.31692/2526-7701.IVCOINTERPDVAgro.2019.0101 }}$

\begin{abstract}
Resumo
$\mathrm{Na}$ ciência do solo existem fatores que podem limitar o uso de softwares diretamente no banco de dados observados, isso ocorre porque os testes de comparação de médias normalmente empregas há uma exigência que os dados sejam homocedásticos e apresente uma distribuição dos resíduos do tipo normal, diante disso os dados precisam de transformações. Para uma análise estatística num teste de comparação de médias adota-se o pressuposto que o conjunto de dados são homocedásticos e que possuem um distribuição normal, aceita-se dados que possuam até leve desvio de distribuição no intervalo -0,5 e 0,5; uma vez que a distribuição é normal quando a média é zero. $\mathrm{O}$ experimento para obtenção do banco de dados foi realizado com arranjo fatorial (3x3) sendo três épocas $(15,30$ e 45 dias) e três níveis de compactação do solo $\left(1,26 ; 1,36\right.$ e $\left.1,46 \mathrm{Kg} \mathrm{dm}^{-3}\right)$, com quatro repetições, o delineamento experimental adotado foi inteiramente casualizado, o feijão (Vigna unguiculata L., Walp) foi plantado em vasos com capacidade para 5 litros seguindo os respectivos níveis de compactação e com avaliação da Altura nas épocas de 15, 30 e 45 após o plantio. Nesse estudo, observa-se que o uso das transformações logarítmicas são essenciais e suas aplicações agronômicas ou não, pois lançando mão desse recurso é possível padronizar os dados para realizar os testes estatísticos clássicos. Conclui-se que A transformação do tipo logarítmica corrigiu a normalidade dos dados, obtendo-se uma distribuição log-normal, segundo teste de Shapiro-Wilk, a homocedascidade dos dados também foi corrigida na transformação logarítmica conforme demostrou o teste de Box-Cox.
\end{abstract}

\footnotetext{
${ }^{1}$ Professor/Engenheiro Agrônomo, Mestrando em Agronomia (Ciência do Solo) e Pós-graduando em Estatística Aplicada, Universidade Estadual Paulista, wanderson.lucena@unesp.br

${ }^{2}$ Mestrando em Ciência do Solo, Universidade Federal do Paraná, andersonagro1 @ hotmail.com

${ }^{3}$ Mestrando em Agronomia (Ciência do Solo), Universidade Estadual Paulista, fariaslucasf@gmail.com

${ }^{4}$ Graduando em Agronomia, Instituto Federal de Pernambuco, hugo.isaac32@gmail.com

${ }^{5}$ Professor/ Especialista, Universidade de Pernambuco, marcianocarvalho148@gmail.com
} 
Palavras-Chave: Estatística, Uso do R, Teste de Box-Cox, Teste de normalidade.

\begin{abstract}
Resumen
En la ciencia del suelo, hay factores que pueden limitar el uso de software directamente en la base de datos observada, esto se debe a que para las pruebas de comparación promedio normalmente empleadas existe el requisito de que los datos sean homoscedastic y tengan una distribución normal de desechos. Ante esto, los datos necesitan transformación. Para un análisis estadístico en una prueba de comparación de medias, se supone que el conjunto de datos es homoscedastic y tiene una distribución normal, se aceptan datos que tienen incluso una ligera desviación de distribución en el rango -0.5 y 0.5 . Pero, para que la distribución es normal cuando la media es cero. El experimento para obtener la base de datos se realizó con arreglo factorial $(3 \times 3)$ con tres estaciones $(15,30$ y 45 días) y tres niveles de compactación del suelo $\left(1.26,1.36\right.$ y $\left.1.46 \mathrm{Kg} \mathrm{dm}^{-3}\right)$. ), con cuatro repeticiones, el diseño experimental fue completamente al azar, el frijol (Vigna unguiculata L., Walp) se plantó en macetas con una capacidad de 5 litros siguiendo los respectivos niveles de compactación y evaluación de altura a los 15, 30 y 45 después de plantar. En este estudio, se observa que el uso de transformaciones logarítmicas es esencial y sus aplicaciones agronómicas o no, porque al usar esta característica es posible estandarizar los datos para realizar las pruebas estadísticas clásicas. Se concluye que la transformación logarítmica corrigió la normalidad de los datos, obteniendo una distribución logarítmica normal, según la prueba de Shapiro-Wilk.
\end{abstract}

Palabras Clave: Estadísticas, uso de R, prueba de Box-Cox, prueba de normalidad.

\begin{abstract}
In the soil science, there are factors that can limit the use of software directly in the observed database; this is because for the average comparison tests normally employed there is a requirement that the data be homoscedastic and have a normal type waste distribution, given this, the data needs transformation. For a statistical analysis in a mean comparison test, it's assumed that the data set is homoscedastic and has a normal distribution, data that have even slight distribution deviation in the range -0.5 and 0.5 are accepted. Since the distribution is normal when the mean is zero. The experiment to obtain the database was performed with factorial arrangement ( $3 \times 3)$ with three seasons (15, 30 and 45 days) and three levels of soil compaction $\left(1.26,1.36\right.$ and $\left.1.46 \mathrm{Kg} \mathrm{dm}^{-3}\right)$. ), with four replications, the experimental design was completely randomized, the bean (Vigna unguiculata L., Walp) was planted in pots with a capacity of 5 liters following the respective compaction levels and height evaluation at 15, 30 and 45 after planting. In this study, it is observed that the use of logarithmic transformations are essential and their agronomic applications or not, because using this feature it is possible to standardize the data to perform the classic statistical tests. It has concluded that: the logarithmic transformation corrected the normality of the data, obtaining a lognormal distribution, according to the Shapiro-Wilk test.
\end{abstract}

Keywords: Statistics, R Usage, Box-Cox Test, Normality Test. 


\section{Introdução}

O software R é uma linguagem e ambiente para calcular estatísticas e gerar gráficos. Este é um projeto General Public License (GNU) o qual é similar à linguagem S e ambiente a qual foi desenvolvida na "Bell Laboratories", formalmente (AT\&T) por John Chambers e colaboradores. O R pode ser considerado uma implantação diferente do S. Existem algumas diferenças importantes, mas muitos dos códigos escritos para o $\mathrm{S}$ rodam sem modificações no R (R CORE TEAM, 2019).

O R fornece uma grande variedade de técnicas estatísticas (modelagem linear e não linear, testes estatísticos clássicos, análise de séries-temporais e etc.) e gráficas, e é altamente extensível. Um dos pontos fortes de R é a facilidade com que bem projetados gráficos para publicações de qualidade pode ser produzidos, incluindo símbolos matemáticos e fórmulas. $\mathrm{O}$ programa $\mathrm{R}$ está disponível como um programa livre (Free Software) sob os termos da Free Software Foundation's GNU (VERZANI, 2004).

A principal dificuldade no uso do R está que normalmente não se conhece lógica nem linguagem de programação e esta dificuldade relacionada a baixa interpretação da lógica estatística e matemática condicionam os estudantes de graduação e pós-graduação a recorrer a softwares privados e/ou de fácil programação, mas sem tanta credibilidade (EKSTROM, 2011).

Na ciência do solo existem fatores que podem limitar o uso de softwares diretamente no banco de dados observados, isso ocorre porque para os testes de comparação de médias normalmente empregas há uma exigência que os dados sejam homocedásticos e apresente uma distribuição dos resíduos do tipo normal, diante disso os dados precisam de transformações. A transformação comumente utilizada é a logarítmica de base neperiana (base número $e=2,71$ ) do valor observado, vale destacar que as transformações não alteram o banco de dados, é apenas um forma estatística e matemática de notação de valor numa escala diferente da observada. Noutros casos é possível fazer transformações utilizando o valor de Lambda ou ainda a raiz quadrada do valor observado (PIMENTEL-GOMES, 2000).

As transformações do tipo log-natural é empregada quando o valor de Lambda do teste de Box-Cox é diferente de um, mas igual a zero. O teste de Box-Cox pertencente ao pacote Mass adequam as os valores observados dentro de um valor predito numa distribuição normal, deixando a variância constante. Após realizar as transformações indicadas por Box e Cox o conjunto de dados deve apresentar homocedásticidade e distribuição normal, para confirmar se há uma distribuição normal nos dados é necessário a aplicação de um teste de normalidade, 
atualmente o mais utilizado é o de Shapiro-Wilk, que apresenta uma estatística "W" e o valor de "p" dentro do intervalo de confiança de 95\% ( $p>0,05)$. Para tal teste, têm-se uma distribuição normal quando p-valor é maior que 0,05 a $5 \%$ de probabilidade ou p-valor maior que 0,01 a $1 \%$ de probabilidade (EKSTROM, 2011).

Diante do exposto, o objetivo geral desse estudo é apresentar um estudo de caso de um banco de dados próprio onde houve a necessidade de transformação de dados para correta avaliação e interpretação dos resultados, utilizando o software livre R.

\section{Fundamentação Teórica}

Para uma análise estatística num teste de comparação de médias adota-se o pressuposto que o conjunto de dados são homocedásticos e que possuem um distribuição normal, aceita-se dados que possuam até leve desvio de distribuição no intervalo -0,5 e 0,5; uma vez que a distribuição é normal quando a média é zero. Porém se os valores médios estiverem dentro do intervalo leve $(-0,5$ e 0,5$)$ os testes são rústicos o suficientes para serem executados sem perda de precisão na inferência estatística (VERZANI, 2004; EKSTROM, 2011).

Para fins de uso didático, nesse estudo, usaremos a interface do R Studio os comandos também podem ser rodados no R comum. A seguir apresentaremos as tabelas 1 e 2 com os símbolos/função e descrição do R (EKSTROM, 2011).

Tabela 1: Operadores matemáticos, funções e descrição do R. Fonte: The R Primer.

\begin{tabular}{|c|c|}
\hline Símbolo/ funções & Descrição \\
\hline 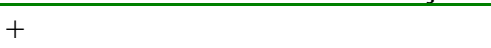 & Adição \\
\hline- & Subtração \\
\hline$*$ & Multiplicação \\
\hline / & Divisão \\
\hline$\wedge$ ou $* *$ & Exponenciação \\
\hline$\% \%$ & Modulus \\
\hline$\% / \%$ & Divisão inteira \\
\hline $\operatorname{abs}(\mathrm{x})$ & Valor absoluto \\
\hline sqrt (x) & Raiz quadrada \\
\hline ceiling $(\mathrm{x})$ & Menor valor não menor que $\mathrm{x}$ \\
\hline floor $(x)$ & Maior inteiro não maoir que x \\
\hline $\operatorname{trunc}(\mathrm{x})$ & Trunca $\mathrm{x}$ descartando as decimais \\
\hline round $(\mathrm{x}$, digits $=0)$ & Arrendonda $\mathrm{x}$ para $\mathrm{n}^{\mathrm{o}}$ de dígitos dec. \\
\hline $\operatorname{signif}(\mathrm{x}$, digits $=6)$ & Arredonda $\mathrm{x}$ para $\mathrm{n}^{\mathrm{o}}$ de dígitos signif. \\
\hline $\cos (x), \sin (x)$ e $\tan (x)$ & Coseno, seno e tangente \\
\hline $\log (\mathrm{x})$ & Logaritmo natural (base $=\mathrm{e})$ \\
\hline $\log (x$, base $=2)$ & Logarítmo com base 2 \\
\hline $\log 10(\mathrm{x})$ & Logarítmo decimal (base $=10$ ) \\
\hline $\exp (x)$ & Função exponencial \\
\hline$\% * \%$ & Multiplicação de matrizes \\
\hline
\end{tabular}


Tabela 2: Símbolos, funções e descrição do R. Fonte: The R Primer.

\begin{tabular}{|c|c|}
\hline Símbolo/função & Descrição \\
\hline mean $(\mathrm{x})$ & Média \\
\hline median $(x)$ & Mediana \\
\hline $\operatorname{sd}(\mathrm{x})$ & Desvio padrão \\
\hline $\operatorname{var}(\mathrm{x})$ & Variância \\
\hline summary (x) & Sumário estatístico \\
\hline $\mathrm{IQR}(\mathrm{x})$ & Intervalo inter-quartílico \\
\hline $\operatorname{cor}(x, y)$ & Correlação entre x e y \\
\hline length $(\mathrm{x})$ & Amplitude de $\mathrm{x}$ \\
\hline $\operatorname{sum}(\mathrm{x})$ & Soma \\
\hline cumsum (x) & Soma acumulativa \\
\hline sort $(\mathrm{x})$ & Ordenação de x \\
\hline $\operatorname{order}(\mathrm{x})$ & Postos de $\mathrm{x}$ \\
\hline $\min (x)$ e $\max (x)$ & Mínimo e maximo de $\mathrm{x}$ \\
\hline quantile $(\mathrm{x})$ & Quantis de $\mathrm{x}$ \\
\hline is.na $(x)$ & Teste para observações perdidas \\
\hline nrow (df) e ncol (df) & $\mathrm{N}^{\circ}$ linhas e colunas no data frame df \\
\hline
\end{tabular}

Essas são algumas operações básicas do R. Contudo para simplificação da leitura será pontuado apenas o estudo das transformações logarítmicas na avaliação de dados.

Um passo importante para avaliação do banco de dados é a montagem do script no $\mathrm{R}$ Studio, dessa forma, visando facilitar a aplicação metodológica do estudo de caso e apresentação dos resultados e sua discussão na fundamentação teórica do estudo estaremos incluindo o script comentado (Script 1).

Script 1: R script de análise fatorial em DIC comentado. Fonte: Própria.

\# Entrando com os dados:

\# Deve-se ter um pasta chamada "R" no disco local C:

dados<-read.table("c://r//fatorial_feijao.txt", h=T) \# Atribuindo o banco de dados dados \# Lendo dados

\# Construir o fator (tratamento) e a resposta (y)

fator $1<$-as.factor(dados\$Epoca)

fator $2<$-as.factor(dados $\$$ Altura)

is.factor(fator1) \# deve ser sempre fator

is.factor(fator2) \# deve ser sempre fator

trat<- as.factor(paste(fator1,fator2,sep="_")) \# Os tratamentos nesse caso é a combinação dos fatores

trat \# Lendo os tratamentos

is.factor(trat) \# Verificando se é um fator

levels(trat) \#Verificando os níveis

$\mathrm{y}<$-dados $\$$ Altura

$\mathrm{y}$

is.numeric(y) \# deve ser sempre numérico 


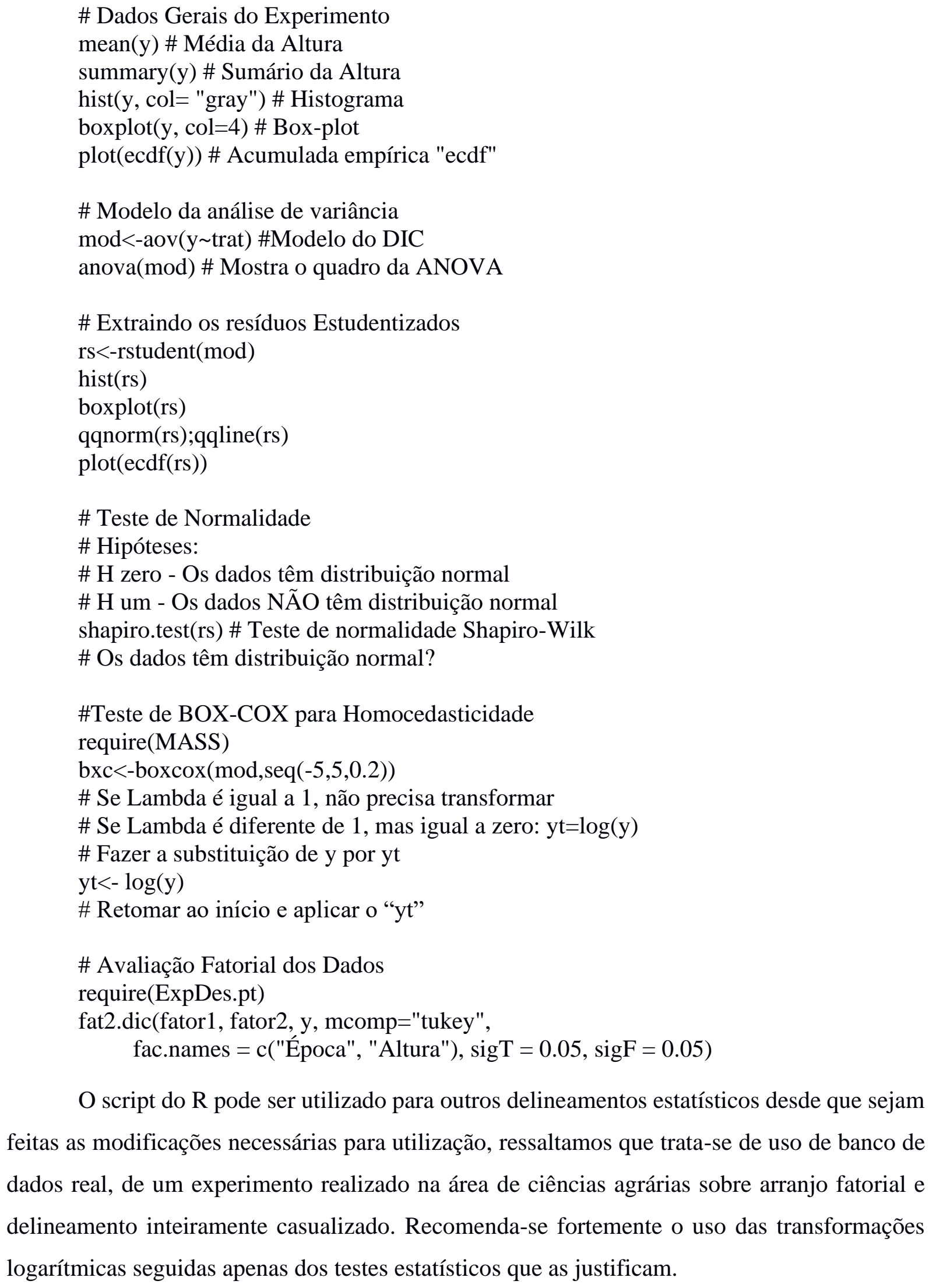
feitas as modificações necessárias para utilização, ressaltamos que trata-se de uso de banco de dados real, de um experimento realizado na área de ciências agrárias sobre arranjo fatorial e delineamento inteiramente casualizado. Recomenda-se fortemente o uso das transformações logarítmicas seguidas apenas dos testes estatísticos que as justificam. 


\section{Metodologia}

O experimento para obtenção do banco de dados foi realizado com arranjo fatorial (3x3) sendo três épocas (15, 30 e 45 dias) e três níveis de compactação do solo $(1,26 ; 1,36$ e 1,46 Kg $\mathrm{dm}^{-3}$ ), com quatro repetições, o delineamento experimental adotado foi inteiramente casualizado, o feijão (Vigna unguiculata L., Walp) foi plantado em vasos com capacidade para 5 litros seguindo os respectivos níveis de compactação e com avaliação da Altura nas épocas de 15, 30 e 45 após o plantio LUCENA et al., 2018). A natural do estudo é quali-quantitativa.

O estudo foi realizado em casa de vegetação no Instituto Federal de Educação, Ciência e Tecnologia de Pernambuco - Campus Vitória de Santo Antão, sob as coordenadas geográficas: $08^{\circ} 05^{\prime} 53^{\prime \prime} \mathrm{S}, 35^{\circ} 17^{\prime} 28^{\prime \prime} \mathrm{W}$, a uma altitude de 156 metros (CPRM, 2005).

No estudo para obtenção do banco de dados há originalmente 12 variáveis, mas nesse estudo de caso de transformações log-natural abordaremos o caso da variável altura. A seguir a tabela 3, apresenta do banco de dados da variável "Altura" nas condições descritas acima.

Tabela 3: Banco de dados da variável Altura nos diferentes níveis dos fatores 1 e 2. Fonte: Própria.

\begin{tabular}{|c|c|c|}
\hline Época & Nível_de_Compactação & Altura \\
\hline 15 & NC1 & 133.00 \\
\hline 15 & NC1 & 130.00 \\
\hline 15 & NC1 & 125.00 \\
\hline 15 & NC1 & 129.33 \\
\hline 15 & NC2 & 118.50 \\
\hline 15 & NC2 & 134.50 \\
\hline 15 & NC2 & 118.50 \\
\hline 15 & NC2 & 134.50 \\
\hline 15 & NC3 & 135.00 \\
\hline 15 & NC3 & 132.50 \\
\hline 15 & NC3 & 135.00 \\
\hline 15 & NC3 & 132.50 \\
\hline 30 & NC1 & 195.00 \\
\hline 30 & NC1 & 165.00 \\
\hline 30 & NC1 & 195.00 \\
\hline 30 & NC1 & 165.00 \\
\hline 30 & NC2 & 122.50 \\
\hline 30 & NC2 & 125.00 \\
\hline 30 & NC2 & 122.50 \\
\hline 30 & NC2 & 125.00 \\
\hline 30 & NC3 & 115.00 \\
\hline 30 & NC3 & 100.00 \\
\hline 30 & NC3 & 100.00 \\
\hline
\end{tabular}




\begin{tabular}{|l|l|l|}
30 & $\mathrm{NC} 3$ & 115.00 \\
\hline 45 & $\mathrm{NC} 1$ & 380.00 \\
\hline 45 & $\mathrm{NC} 1$ & 380.00 \\
\hline 45 & $\mathrm{NC} 1$ & 315.00 \\
\hline 45 & $\mathrm{NC} 1$ & 315.00 \\
\hline 45 & $\mathrm{NC} 2$ & 295.00 \\
\hline 45 & $\mathrm{NC} 2$ & 255.00 \\
\hline 45 & $\mathrm{NC} 2$ & 255.00 \\
\hline 45 & $\mathrm{NC} 2$ & 295.00 \\
\hline 45 & $\mathrm{NC} 3$ & 250.00 \\
\hline 45 & $\mathrm{NC} 3$ & 265.00 \\
\hline 45 & $\mathrm{NC} 3$ & 250.00 \\
\hline 45 & $\mathrm{NC} 3$ & 265.00 \\
\hline
\end{tabular}

NOTA: Para entrada de dados no R, recomendamos o não uso de acentos e/ou espaços entre palavras, fazer uso do ponto com separador decimal e salvar o arquivo no formato (.txt) separado por tabulações.

O primeiro passo para verificação é salvar o banco de dados no formato (.txt) e separado por tabulações, copiar e colar o R script no R Studio e seguir os procedimentos descritos nessa metodologia.

$1^{\text {o }}$ Passo: Copiar e colar o R script no R Studio, após selecionar as duas primeiras linhas de comando (linhas 3 e 4) e clicar em RUM, ver imagem abaixo:

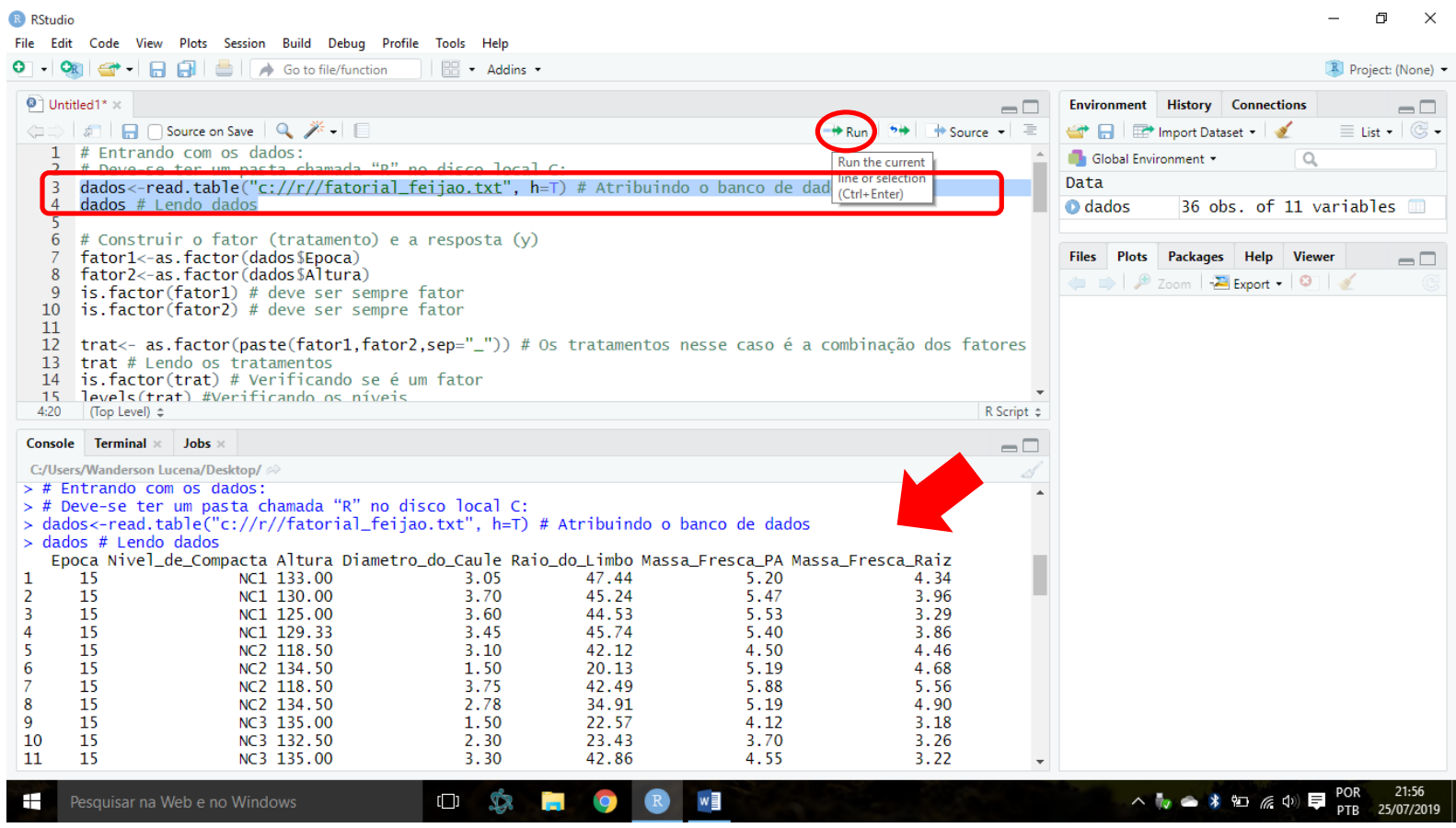

$2^{\circ}$ Passo: No R Studio selecionar as linhas de 06 a 19 e rodar (clicar em rum ou dá o comando CTRL + Enter no teclado): 


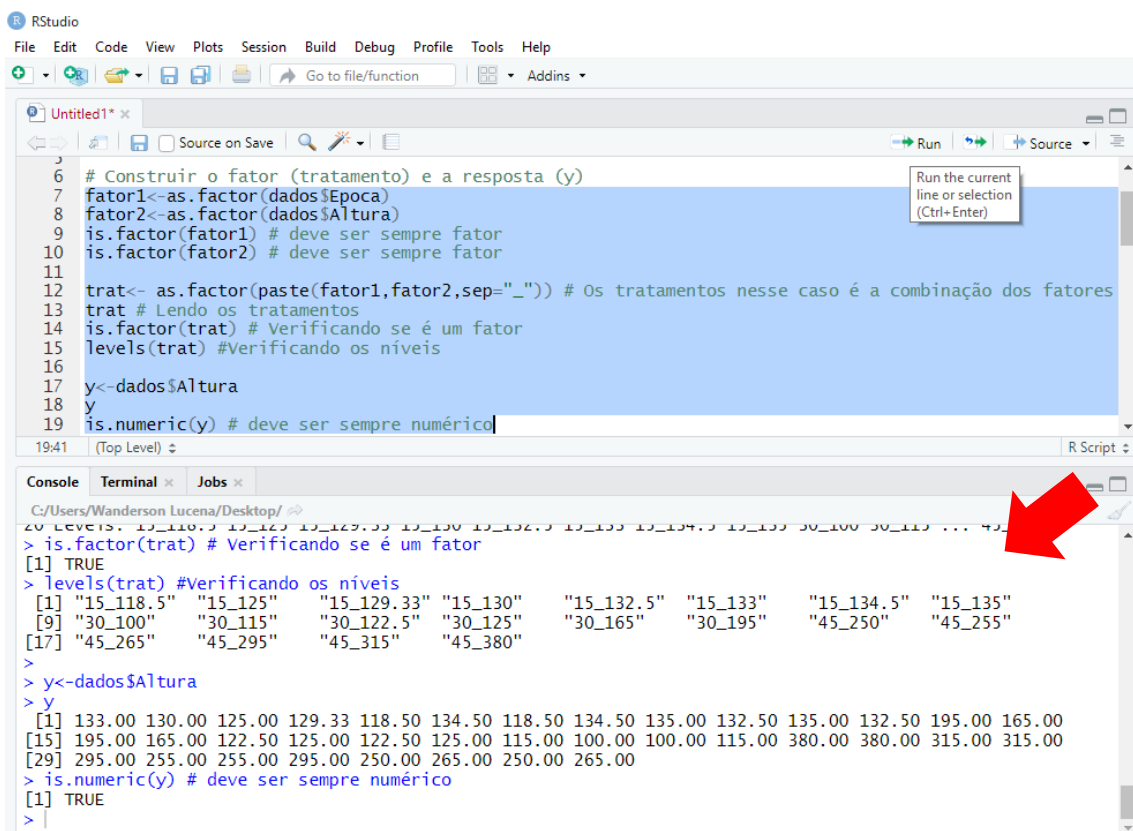

$-\quad 0 \times$

$3^{\text {o }}$ Passo: Analisando os dados gerais, assim como histograma, box-plot e acumulada empírica, recomendamos que faça linha por linha para visualizar os gráficos na janela "Plots", em destaque na imagem abaixo as informações do box-plot (estatística descritiva):

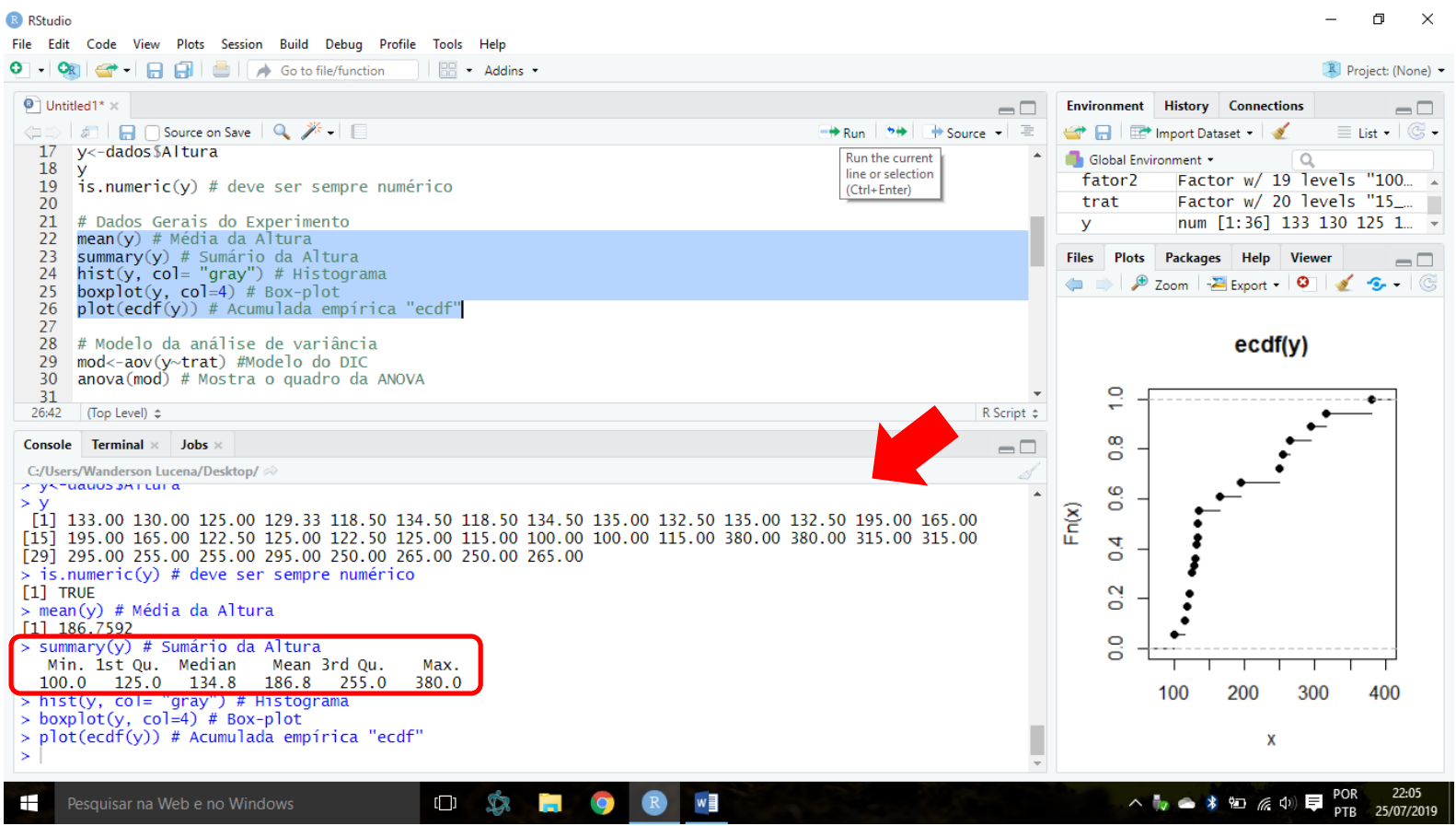

$4^{\circ}$ Passo: Executar as linhas selecionadas como na imagem abaixo, assim estará sendo realizado o quadro da análise de variância e do comportamento do resíduo: 

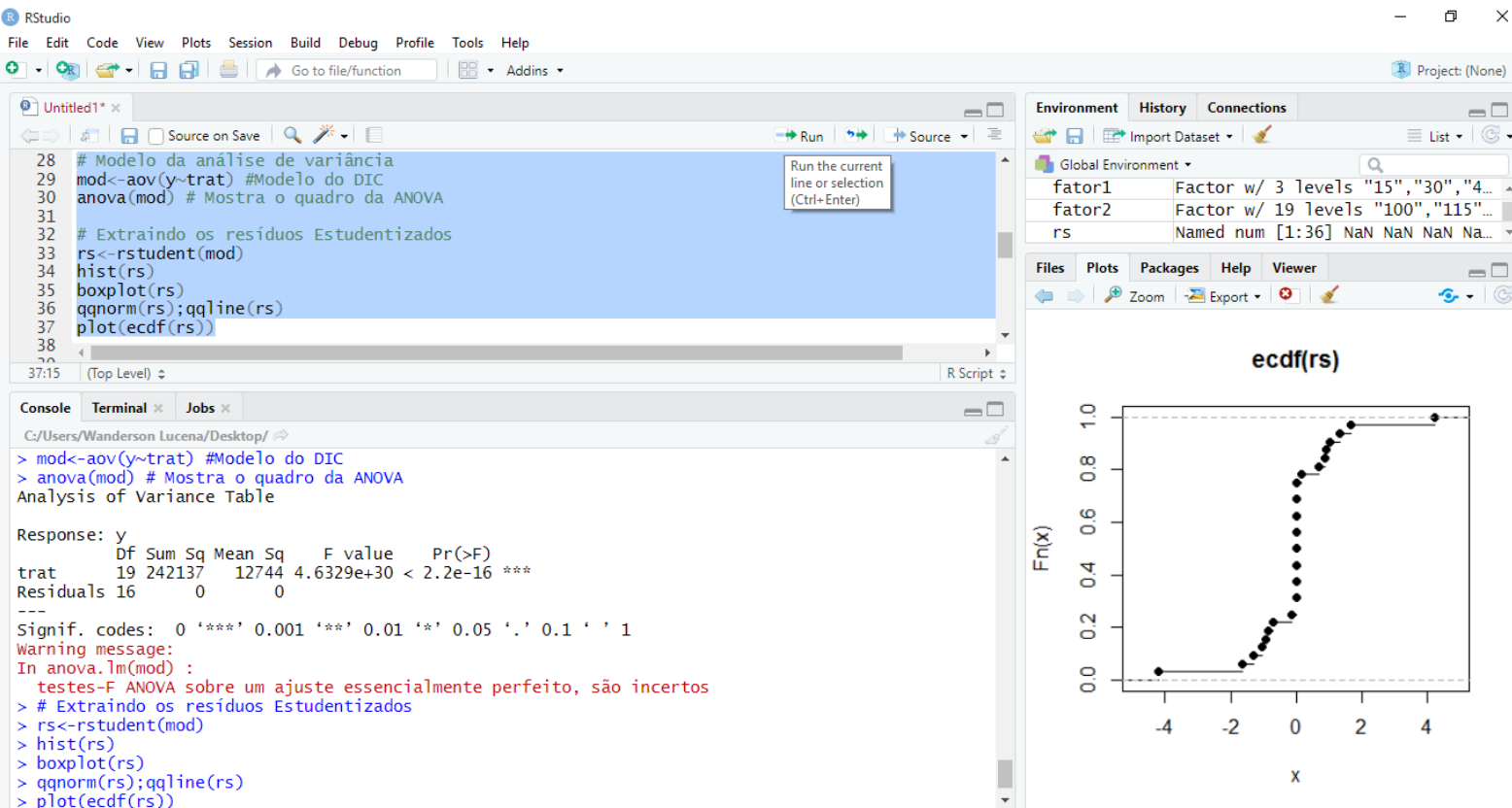

* Pesquisar na Web e no Windows

$5^{\circ}$ Passo: Rodar a linha número 43 no R Script e observar a resposta no console do R Studio, de acordo com os dados a variável "Altura" não possui um distribuição normal para $p$ valor $=5,421 \times 10^{-5}$, segundo teste de normalidade de Shapiro-Wilk:

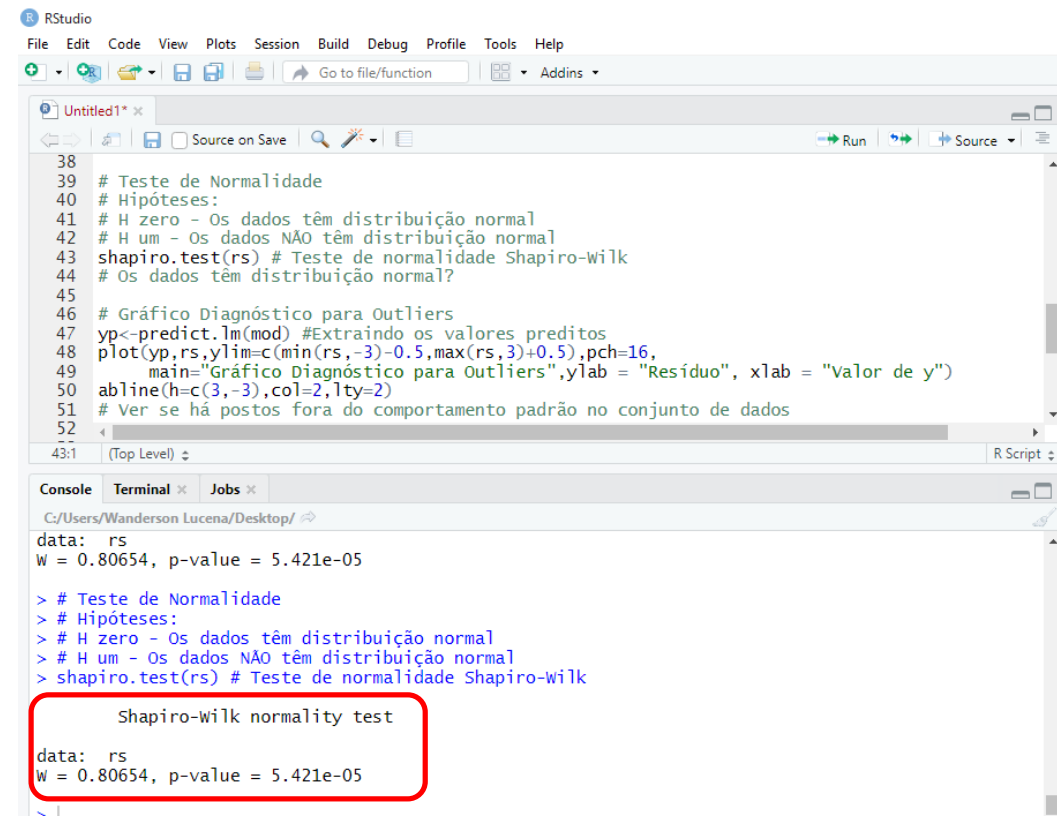

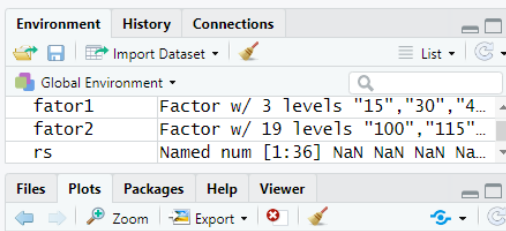

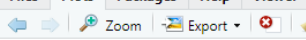

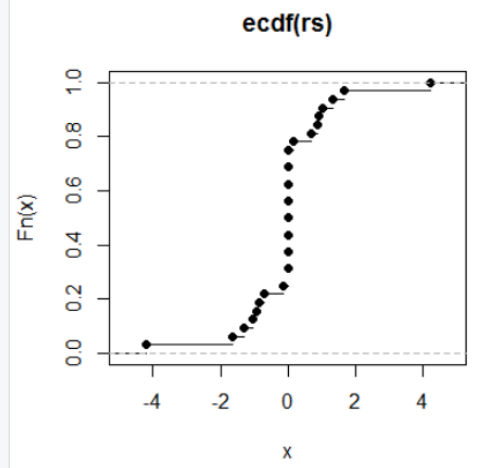

* Pesquisar na Web e no Windows $\quad\left[\begin{array}{llllll} & \text { a } & \text { B } & \text { wl }\end{array}\right.$

$6^{\circ}$ Passo: Realizar o teste de homocedacidade de Box-Cox, conforme ilustração abaixo: 


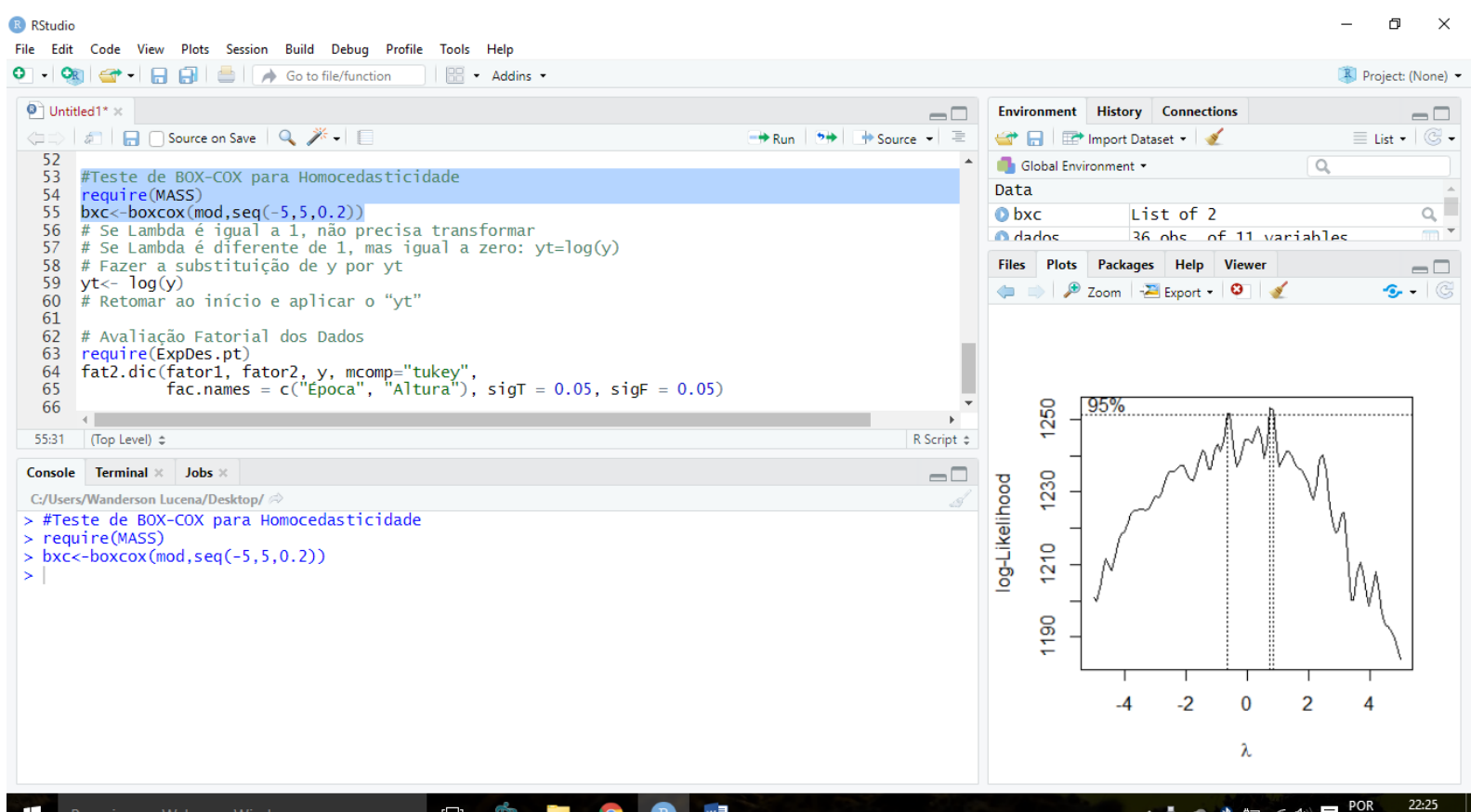

Observe que segundo o teste de Box-Cox o intervalo de confiança não inclui o número 1, mas inclui o zero, indicando assim uma transformação dos dados, sendo [Ln $(y+K)]$, onde K é uma constante, no R Studio, basta fazer e atribuir o $[\log (\mathrm{y})]$.

$7^{\circ}$ Passo: Aplicar ao yt o log de y, em seguida voltar ao início da análise e aplicar yt para todo y, verificar a normalidade e homocedacidade do dados para só assim realizar a análise fatorial dos dados.

\section{Resultados e Discussão}

Nesse estudo, observa-se que o uso das transformações logarítmicas são essenciais e suas aplicações agronômicas ou não, pois lançando mão desse recurso é possível padronizar os dados para realizar os testes estatísticos clássicos. Verificou-se ainda que não há alterações numéricas no valor observado no experimento, os dados podem ser consultados no trabalho de Lucena et al. (2018); isso ocorre porque as transformações desse tipo é apenas uma notação matemática de reescrever o número observado na escala de log-neperiano, deixando assim os valores dentro de uma distribuição de comportamento normal (PIMENTAL-GOMES, 2000, 2011).

A utilização de programação e estatística no R facilita o uso dessa correção, bem como a comodidade de está utilizando um software estatístico mundialmente aceito e de licença gratuita. Vale lembrar que existem outros tipos de transformações que são comumente usadas na estatística, para saber qual é mais apropriada ao estudo, recomenda-se fazer uso do teste de 
Box-Cox, os comandos para realização de tal teste encontra-se na metodologia desse trabalho.

É imprescindível saber se o banco de dados em questão suporta correção ou não, caso contrário a transformação não atenderá seus objetivos, conforme mencionado nesse trabalho.

$\mathrm{Na}$ ciência do solo ou em ciências naturais e da terra os dados observados dificilmente apresentam comportamento normal e homocedástico, as transformações tem como objetivo a padronização dos dados numa escala para melhor utilização dos testes estatísticos clássicos, como, análise de variância e comparação de médias.

Vale destacar que em estatística é fundamental na análise de dados provenientes de quaisquer processos onde exista variabilidade, no entanto, desvios de variabilidade interna ou entre tratamentos de comportamento não-normal não podem ser identificados em testes estatísticos, por isso, justifica-se a utilização das transformações para correção do banco de dados o qual deseja-se avaliar e fazer a inferência estatística.

Em suma, atendendo o objetivo desse trabalho que foi de demostrar que a aplicação das transformações logarítmicas num caso prático de experimento possui resultado satisfatórios, como é esperado já que na literatura em estatística aplicada é amplamente usada e discutida.

De acordo com dados avaliados e a discursão de Lucena et al. (2018), pode inferir que no experimento o comportamento do dados foram: Aos 30 Dias Após Semeadura (DAS) houve diferença significativa no nível de compactação 1 quando comparado com os níveis 2 e 3, esses não diferiram entre si, mas foram limitantes à cultura. Esses resultados se repetiram aos 45 DAS. Assim até 15 DAS nenhum dos três níveis de compactação estudados interferiu no crescimento do feijoeiro, no entanto, aos 30 e 45 DAS os níveis de compactação 2 e 3 foram limitantes ao crescimento da planta. Conclui-se que o feijão de corda possui limitação do crescimento em solos com níveis de compactação superiores a $1,36 \mathrm{~kg} \mathrm{dm}^{-3}$ a partir dos 30 DAS (MANTOVANELLI, 2017).

\section{Conclusões}

A transformação do tipo logarítmica corrigiu a normalidade dos dados, obtendo-se uma distribuição log-normal, segundo teste de Shapiro-Wilk;

Homocedascidade dos dados também foi corrigida na transformação logarítmica conforme demostrou o teste de Box-Cox;

Após as transformações o banco de dados comportou de forma correta a aplicação da análise de variância e dos testes de comparação de médias. 
No trabalho, os autores apontam um limitação no crescimento do feijão, quando avaliado o parâmetro altura, portanto, falta a relação com demais parâmetros do estudo para confirmar essa limitação.

Por outro lado, estudos de 2017 já evidenciaram essa limitação de crescimento em outras culturas também em áreas de zona da mata, reforçando assim a afirmação anteriormente citada.

\section{Referências}

CUNHA, E. Q.; STONE L. F.; FERREIRA, E. P. B.; DIDONET, A. D. MOREIRA, J. A.A.; LEANDRO, W. M. Sistemas de preparo do solo e culturas de cobertura na produção orgânica de feijão e milho II - atributos biológicos do solo. Revista Brasileira de Ciência do Solo, Viçosa, v. 35, n. 2, p. 603-611, 2011.

CRAWLEY, M. J. The R book. London, Wiley, 2007.

CPRM - Serviço Geológico do Brasil. Projeto cadastro de fontes de abastecimento por água subterrânea estado de Pernambuco: diagnóstico do município de Lagoa de Itaenga. Recife: CPRM/PRODEEM, 2005. Disponível em: http://www.cprm.gov.br/rehi/atlas/pernambuco/relatorios/LDIT093.pdf>. Acesso em: 05 de maio de 2018.

EMPRESA BRASILEIRA DE PESQUISA AGROPECUÁRIA (EMBRAPA). Embrapa Solos. Ministério da Agricultura, Pecuária e Abastecimento (MAPA). Sistema brasileiro de classificação de solos. 3. ed. rev. e amp. Brasília, 2013. 353 p.

EMPRESA BRASILEIRA DE PESQUISA AGROPECUÁRIA (EMBRAPA). Centro Nacional de Pesquisa de Solos. Manual de métodos de análise de solo. 3.ed. Rio de Janeiro, 2017. 212 p.

EKSTROM, C.T. The R Prime. Chapman \& Hall/CRC, 2011.

FILGUEIRA, F. A. R. Novo manual de olericultura: agrotecnologia moderna na produção e comercialização de hortaliças. 3. ed. Viçosa: Ed. UFV, 2008.

LUCENA, W. B.; SILVA, H. I; SILVA, A. L.; SILVA NETO, J. M.; SILVA, K. M.; BEZERRA, S. A. Efeitos da compactação do solo no crescimento do feijão de corda. In: XVIII CONGRESSO PARANAENSE DE ENGEHEIROS AGRÔNOMOS. 2018. Guarapuava-PR. Anais eletrônicos... $\quad$ Disponível em <https://docs.wixstatic.com/ugd/6ded74_138d248b1c6f4b9bacdc51cd3b9284bc.pdf>. Acesso em 25 de Nov. de 2018.

MAINDONALD, J. \& BRAUN, J. Data analysis and graphics using R: an example-based approach. Cambridge, $2^{a}$ ed., 2006.

MANTOVANELLI, B. C. Distribuição espacial de atributos físicos e quantificação de poros via tomografia computadorizada em solo cultivado com cana-de-açúcar sob 
diferentes manejos. 2017. 97 f. Dissertação (Mestrado em Ciência do Solo) - Universidade Federal Rural de Pernambuco, Recife, 2017.

MURREL, P. R Graphics. London, CRC, 2006.

PETERnELli, L. A., MELlO, M. P. Conhecendo o R Uma Visão Estatística, Viçosa, UFV editora, $1^{\mathrm{a}}$ ed., 2011.

PIMENTEL-GOMES, F. Curso de Estatística Experimental. Piracicaba, Editor: F. PimentelGomes, $14^{\mathrm{a}}$ ed., 2000.

R CORE TEAM (2019). R: A language and environment for statistical computing. $R$ Foundation for Statistical Computing, Vienna, Austria. URL https://www.R-project.org/.

VERZANI, J. Using R for Introductory Statisticas. London, Chapman \& Hall/CRC, 2004. 